\title{
Myocardial Infarction in the Absence of Obstructive Coronary Artery Disease (MINOCA): a Review of the Present and Preview of the Future
}

\author{
Soheila Talebi ${ }^{1}$ - Preeti Jadhav ${ }^{1}$ - Jacqueline E. Tamis-Holland ${ }^{1}$ \\ Accepted: 12 April 2021 / Published online: 6 July 2021 \\ (C) The Author(s), under exclusive licence to Springer Science+Business Media, LLC, part of Springer Nature 2021
}

\begin{abstract}
Purpose of Review The syndrome of myocardial infarction in the absence of obstructive coronary artery disease (MINOCA) is not uncommon and has multiple potential coronary etiologies. With the use of more sensitive cardiac biomarkers and advanced cardiovascular imaging, MINOCA presentations have gain increasing attention among researchers and cardiologists. Despite the presence of a myocardial infarction and elevated future risk, many patients are sent home with little or no cardio-protective treatment and no explanation for their symptoms. In this review, we emphasized the importance of MINOCA treatment based on the underlying etiology.

Recent Findings As there are multiple pathophysiological mechanisms potentially involved in MINOCA, it should be considered a working diagnosis until there is a better understanding regarding the underlying cause. It is critical to use multimodality imaging when treating patients with MINOCA to help determine the underlying etiology and rule out mimics of MINOCA, so that therapies appropriate to the etiology can be provided.

Summary A more systematic approach to managing patients with MINOCA should result in better treatment and an improved prognosis for these patients.
\end{abstract}

Keywords MINOCA $\cdot$ Multimodality $\cdot$ Optical coherence tomography $\cdot$ Cardiac magnetic resonance $\cdot$ Coronary computed tomographic angiography $\cdot$ Echocardiography $\cdot$ Myocardial infarction $\cdot$ Women

\section{Introduction}

Myocardial infarction in the absence of obstructive coronary artery disease (MINOCA) is defined by a presentation consistent with an acute myocardial infarction (AMI; as outlined by the Universal Definition for Acute Myocardial Infarction [1]), the lack of obstructive coronary artery disease $(>=50 \%$ stenosis), and no other explanation for the clinical presentation at the time of coronary angiography $[2 \bullet \cdot]$. MINOCA accounts

Topical Collection on Women and Ischemic Heart Disease

Soheila Talebi

Soheila.Talebi@mountsinai.org

Preeti Jadhav

Preeti.Jadhav@mountsinai.org

Jacqueline E. Tamis-Holland

Jacqueline.Tamis-Holland@mountsinai.org

1 Mount Sinai Heart, Icahn School of Medicine at Mount Sinai, 1111 Avenue New York, Amsterdam, NY 10025, USA for 5\%-15\% of patients with AMI [3] and is associated with a $5 \% 1$-year mortality. The pathogenesis of MINOCA is varied and may include coronary etiologies such as atherosclerotic plaque rupture, plaque erosion with thrombosis, coronary artery dissection, epicardial coronary vasospasm or microvascular spasm, coronary embolization, or a combination of mechanisms. Additionally, nonischemic causes may often mimic MINOCA, including Takotsubo syndrome, acute myocarditis, and pulmonary embolism. For these reasons, it is important to perform a complete assessment of patients presenting with suspected MINOCA so that a correct diagnosis might be identified.

Most of the pathogenetic mechanisms leading to MINOCA are well recognized as unusual causes of AMI, but raising awareness of this condition has led to increased diagnosis of these etiologies in the last few years, particularly in young-tomiddle-aged women presenting with AMI. This is especially important because MINOCA disproportionately affects females, with a prevalence of approximately $10 \%-15 \%$ of females compared to $3 \%-4 \%$ among males with AMI $[4,5]$. In the absence of obstructive coronary artery disease, clinicians 
often fail to correctly diagnose the AMI, with the result that patients are mistakenly reassured and discharged without medical therapy, without a true diagnosis or treatment [6]. To define the underlying cause, scientific statements and guidelines recommend multimodality imaging in MINOCA $[2 \bullet \bullet, 7,8]$. A recent review [9] highlights the importance of such imaging to uncover all cases of MINOCA. In the current paper, we review the major coronary etiologies of MINOCA, highlight newer data supporting multimodality imaging in patients with suspected MINOCA, and provide some case examples.

\section{A Review of MINOCA Etiologies}

\section{Coronary Plaque Disruption}

Type I AMI are infarctions resulting from plaque rupture, plaque erosion, or calcified nodules, with or without residual thrombus at the site of the culprit lesion. Impairment of the vascular endothelium leads to thrombosis and results in partial or complete obstruction of the epicardial coronary artery lumen or platelet micro-emboli to the microvasculature. If the degree of stenosis of the coronary artery is $<50 \%$, or if there are emboli to the distal microvasculature alone, the patients will be found to have nonobstructive disease on angiography (MINOCA).

Rupture or erosion of an atherosclerotic plaque may occur in areas of the vessel that appear normal or have a minimal degree of atherosclerosis on conventional angiography, and therefore additional assessment is often indicated. Plaque rupture is best diagnosed by intracoronary imaging, with OCT the preferred modality as it can detect plaque erosion as well as rupture. Studies have shown that approximately $38-40 \%$ of patients with MINOCA have some evidence of plaque disruption, including plaque rupture, erosion, or calcified nodules when intracoronary imaging is performed $[10,11,12 \bullet \cdot]$. Among patients with MINOCA and plaque disruption, cardiac magnetic resonance (CMR) imaging may show large areas of myocardial edema with or without small areas of necrosis, suggesting that flow was temporarily compromised in a larger vessel. Both spontaneous coronary thrombolysis and vasospasm can lead to this appearance and cannot be distinguished without coronary provocative testing. A smaller, well-defined area of late gadolinium enhancement (LGE) subtended by a smaller vessel may be shown by CMR imaging, indicating that the embolization of atherothrombotic debris from the disruption site is the most likely mechanism of myocardial necrosis $[11,12 \bullet \bullet]$.

Current guidelines recommend medical treatment of plaque rupture with MINOCA in a similar manner to plaque rupture with obstructive coronary artery diseaese [9]. Therefore, for patients with confirmed plaque disruption and
MINOCA, dual antiplatelet therapy can be prescribed for 1 year followed by lifetime single antiplatelet therapy. Because disruption occurs in the context of nonobstructive coronary artery disease, statin therapy is also recommended, even if there is only a minor degree of atherosclerosis [8].

\section{Spontaneous Coronary Artery Dissection}

Spontaneous coronary artery dissection (SCAD) is an uncommon cause of AMI characterized by a nontraumatic and noniatrogenic separation of the layers of the coronary arterial wall resulting from intramural hematoma. This intramural hematoma will compress the true lumen and lead to a decrease in arterial blood flow. There are several types of SCAD described in the literature [13]. The tapering appearance of the arterial lumen that is seen in type II SCAD can often be misinterpreted as a nonobstructive lesion, resulting in MINOCA.

SCAD is present in approximately $20 \%$ of patients with MINOCA [4]. SCAD is more common in women than that in men and is observed in approximately one quarter of women $<50$ years of age with acute coronary syndromes [14]. SCAD is associated with younger age, female gender, fibromuscular dysplasia (FMD), pregnancy, and the peripartum period in the absence of conventional risk factors for coronary heart disease. A combination of factors that include sex hormonal fluctuations, genetics, underlying arteriopathies, and physical or emotional precipitants are likely to influence SCAD [15].

Most cases of SCAD are diagnosed on coronary angiography which often depicts a radiolucent flap, dual lumen, and contrast staining. However, type II SCAD may not be immediately apparent on coronary angiography. The presence of a rapidly tapering vessel with tortuous, "corkscrew"-like vessels maybe a clue to this diagnosis. IVUS or OCT can be useful in confirming SCAD, but due to the potential risk of propagating the dissection or entering the false lumen with wire manipulation and device advancement, intracoronary imaging should be selectively used only when imaging would alter the plan of treatment. Coronary CT angiography can be a useful tool when coronary angiography is not clearly diagnostic but does not have the spatial resolution to distinguish intramural hematoma from noncalcified plaque [16].

Conservative management is preferred over coronary revascularization when treating SCAD [15]. Coronary revascularization might be considered in patients with hemodynamic instability or with ongoing ischemia in the presence of a totally occluded vessel or slow flow. Due to the fragility of the vessel wall, PCI can be challenging and is associated with high failure rates, with potential propagation of the hematoma beyond the site of the intervention [17]. Routine recurrent coronary angiography to assess SCAD healing should be 
avoided during follow-up, as the benefits do not outweigh the potential risks of iatrogenic dissection [18].

The role of antiplatelet therapy in SCAD remains controversial. Despite the concern that dual antiplatelet therapy (DAPT) may further expand the intramural hematoma, expert consensus is that it could be beneficial, since the intimal tear in SCAD can be prothrombotic [15]. Beta-blockers are useful in cases of SCAD as they have been shown to decrease the risk of recurrence [19]. Additionally, due to their cardio-protective mechanisms, they remain part of the guideline recommendations for patients with AMI $[20,21]$. Since SCAD is not a result of atherosclerosis, lipid lowering therapy with statins should only be prescribed if there is concomitant atherosclerosis or pre-existing dyslipidemia. A small retrospective study showed potentially higher SCAD recurrence with statins [15].

\section{Coronary Vasospasm}

Epicardial coronary artery vasospasm is a transient and reversible vasoconstriction of the major epicardial coronary artery that can result in AMI. Coronary vasospasm therefore is a common cause of MINOCA. Its prevalence will vary across race, with a larger proportion of coronary vasospasm seen in Asian populations. In one series, coronary vasospasm was reported in $40 \%$ of patients with MINOCA undergoing provocative spasm testing [22]. In contrary to the other phenotypes of MINOCA, coronary vasospasm has a male predominance and is associated with higher rates of smoking and alcohol consumption [23].

Patients usually present with typical chest pain at rest or while asleep, associated with transient ST segment elevations, or at times ST segment depressions and $\mathrm{T}$ wave inversion which resolves spontaneously or following the administration of nitrates [24]. The diagnosis of coronary vasospasm is made if there are either spontaneous episodes with ECG changes, reversed with NTG, or if provocation results in chest pain, ECG changes, or angiographic evidence of epicardial coronary vasospasm. Because coronary vasospasm is a transient functional abnormality, spasm provocation plays a key role in diagnosing this condition. Provocation can be performed with exercise or hyperventilation or through pharmacologic mechanisms administered in the cath laboratory under controlled circumstances. Provocative testing with acetylcholine is the gold standard to demonstrate reversible coronary obstruction and demand supply mismatch of coronary blood flow [25]. Provocative testing is considered positive for coronary vasospasm when there is demonstration of the following findings: (1) reproduction of usual chest pain syndrome; (2) ischemic EKG changes; and (3) $>90 \%$ vasoconstriction on angiography [26].

Patients with vasospastic angina can be treated with calcium channel blockers and nitrates [26]. Treatment with calcium channel blockers has been shown to be an independent predictor of survival. In cases of refractory angina, two calcium channel blockers with different mechanisms of action may be useful [27]. Due to the potential increase in vascular tone associated with routine beta blocker use, these drugs are not useful for treating coronary artery vasospasm [26]. Patients with coronary vasospasm often have some degree of underlying atherosclerotic plaque, and therefore statin therapy may be indicated.

\section{Coronary Thrombus or Embolism}

Coronary embolism is the underlying cause of 3\%-7\% of acute coronary syndromes [28]. In patients with MINOCA, one should suspect either coronary embolism or thrombosis to an epicardial coronary artery with partial resolution at the time of angiography or embolism or thrombosis to the distal microvasculature, if there is a known underlying source (i.e., left atrial or ventricular thrombus) or if there is a suspicion of a hypercoagulable state. Coronary embolism maybe be direct (from the left ventricle, aortic or mitral valves, or the left atrial appendage), paradoxical (from the venous circulation through a patent foramen ovale), or iatrogenic (following cardiac intervention). Certain genetic thrombophilic disorders like protein $\mathrm{C}$ and $\mathrm{S}$ deficiency, factor $\mathrm{V}$ Leiden mutation, and antiphospholipid syndrome are more prevalent in MINOCA than the general population in multiple observational studies and registries [28, 29]. Pregnancy is a known procoagulant state, and the effect of estrogen and oral contraceptives on precipitating thrombosis as well as AMI are well documented in literature [30].

The diagnosis of an embolic event or hypercoagulable disorder is largely based on the clinical presentation and presence of risk factors for such conditions. In situations where there is a "hazy" appearance on coronary angiogram, intracardiac imaging with IVUS or OCT can help differentiate spontaneous coronary thrombosis or embolization from other etiologies of MINOCA with plaque disruption. Echocardiography, TEE, and bubble studies are useful to find the source of emboli. Thrombophilia in MINOCA is uncommon but selected patients may benefit from further screening $[2 \cdot \bullet]$.

Treatment options are based on the specific etiology and precipitating factors for coronary embolization. Anticoagulation is indicated in cases of embolization of thrombus from a remote site (i.e., atrial fibrillation or left ventricular thrombus). In selected cases of suspected paradoxical embolism, a definitive surgical closure of a PFO or ASD may help prevent recurrent episodes of AMI [31]. In patients with disorders of coagulation, the decision to treat with anticoagulant or antiplatelet therapies should generally be made with consultation from a hematologist. 


\section{COVID-19 Infection}

In severe acute respiratory syndrome coronavirus 2 infection (COVID-19), a significant number of patients develop chest pain or EKG changes and are found to have an elevation of cardiac troponin suggestive of myocardial injury. In these patients, myriad potential mechanisms may account for this presentation, including myocardial injury from nonischemic mechanisms, type I AMI due to an increased propensity for plaque rupture, or type II AMI resulting from severe diffuse thrombosis, supply demand mismatch, or coronary vasospasm $[32 \bullet \cdot 33,34]$. There is growing evidence that a widespread hypercoagulable state develops as a systemic manifestation of severe COVID-19 infection [35]. The data from various observational studies supports increased incidence of hypercoagulable state in these patients mainly due to increased level of inflammatory markers like C-reactive protein, fibrinolysis, and presence of antiphospholipid antibodies. In an autopsy study of patients dying of COVID-19, cardiac microthrombi was the most common pathological cause of myocyte necrosis and differed in composition from coronary thrombi in ST-segment elevation acute myocardial infarction (STEMI) patients whether they were SARSCoV-2 positive or negative [36]. Patients with STEMI are found to have increased burden of thrombus as well. In a small observational study, it was noted that patients with STEMI and COVID-19 infection had increased incidence of MINOCA due to thrombotic occlusion and distal embolization [37]. Finally, recently published case reports and biopsy findings suggest that SARS-CoV-2 can cause direct infection or immune-mediated widespread inflammation of endothelial cells leading to endothelitis [38]. In patients with COVID-19, patients with coronary etiologies including MINOCA, spontaneous thrombosis, or coronary vasospasm should be distinguished from other forms of myocardial injury including myocarditis. If there is a suspicion of thrombosis, intravascular imaging with OCT or IVUS can help differentiate patients with pro-thrombotic etiologies from those with traditional atherosclerotic plaque rupture. Cardiac MR maybe helpful in distinguishing conditions causing MINOCA with nonischemic mechanisms of myocardial injury [34]. Endomyocardial biopsy is not routinely recommended, as virus and lymphocytic infiltration is very rarely observed in postmortem samples, suggesting that the yield would be low in vivo [39].

There is no formal consensus on the optimal treatment of patients with MINOCA resulting from COVID-19 infection [40]. Most importantly, treatment would depend on the underlying cause. In cases with evidence of thrombosis, therapeutic anticoagulation would be a logical treatment, although in the absence of thrombosis, the INSPIRATION trial demonstrated that it should not be used for routine prophylaxis in intensive care patients with COVID-19 [41], with decreased thrombotic complications balanced by increased bleeding events [39, 40, 42, 43]. Randomized trials of anticoagulation in the inpatient and outpatient setting are ongoing. For this reason, it is important to individualize care and update protocols with changing data in this unique population of patients with MINOCA.

\section{A Preview of the Future of MINOCA: Multimodality Imaging}

Expert consensus statements and guidelines have emphasized the importance of multimodality imaging in patients with suspected MINOCA [2••, 7, 8]. The overall goals are to confirm the location and extent of myocardial necrosis, to exclude nonischemic mechanisms, and to diagnose the coronary etiology to direct appropriate therapy. This includes a careful review of angiographic and echocardiography findings, followed by dedicated intracoronary imaging for the majority of patients in whom the etiologic cause of MINOCA is unclear, and subsequent use of cardiac MRI. While this is intuitive, until recently there was limited data to support this systematic approach to evaluating patients with MINOCA.

The Women's Heart Attack Research Program (HARP) was a multicenter prospective study designed to perform dedicated diagnostic imaging with OCT (performed at the time of diagnostic angiography) and cardiac MRI (performed within 1 week of enrollment) in women with MINOCA to understand the degree of myocardial involvement and investigate the underlying cause $[12 \bullet \cdot$ ]. A total of 301 women with MINOCA were enrolled at 16 sites of whom 145 had interpretable OCT imaging. A definite culprit lesion was found in $46.2 \%$ of patients undergoing OCT. This included plaque disruption in $40 \%$ of patients, thrombus in $3.4 \%$ of patients, intimal "bumping" (suggestive of coronary vasospasm) in $2.1 \%$ of patients, and SCAD in one patient. Cardiac MRI was interpretable in 116 of the 145 patients with adequate OCT imaging. Among patients undergoing MRI with interpretable imaging, $32.8 \%$ had late gadolinium enhancement on cardiac MRI consistent with necrosis. Regional injury and/or abnormal excess of intracellular volume was reported in $20.7 \%$ of cases. Myocarditis was found in $14.7 \%$ of patients. Takotsubo syndrome was noted in $3.4 \%$, and $2.6 \%$ of patients had a nonischemic cardiomyopathy. Taken together, a clinical diagnosis was identified in $85 \%$ of women with MINOCA who had multimodality 
imaging with OCT and MRI. This research gives support to the management algorithms proposed by expert consensus statements and demonstrates that intracoronary imaging and MRI are feasible and have the potential to guide secondary prevention therapy.

In another smaller study [44], patients with suspected MINOCA were subject to a standard diagnostic algorithm which included provocative testing for coronary vasospasm followed by cardiac MRI and OCT imaging. In this study of 116 patients, $29 \%$ were found to have epicardial coronary vasospasm and therefore did not undergo further intracoronary imaging. Of the remaining patients, $66 \%$ had both OCT and cardiac MRI, and $73 \%$ of these patients had interpretable studies. A clear culprit lesion was identified on OCT in $80 \%$ of patients (plaque rupture $35 \%$; calcific nodule $2.5 \%$; plaque erosion $30 \%$; lone thrombus $7.5 \%$; and SCAD $5 \%$ ). MRI identified findings supportive of infarction in $77.5 \%$ of patients, and the combination of OCT imaging and CMR provided a diagnosis in all patients undergoing multimodality imaging.

\section{Case Examples}

\section{Case 1: MINOCA Secondary to Paradoxical Emboli}

A 53-year-old woman with a past medical history of hypertension presented with chest pain. A 12-lead electrocardiogram showed normal sinus rhythm with nonspecific STsegment deviations. Cardiac troponin peaked at $20 \mathrm{ng} / \mathrm{mL}$. An echo showed mild left ventricle hypertrophy with preserved left ventricle systolic function. Coronary angiography showed normal epicardial coronary arteries. Cardiac MRI revealed a focal transmural myocardial infarction at the mid- ventricular portion of the anterolateral wall (Fig. 1A and B), a small atrial septal defect, and a Qp/Qs ratio of 1.3. Transesophageal echo confirmed a small secundum atrial septal defect (Fig. 1C). A diagnosis of MINOCA secondary to paradoxical emboli was made and the patient was referred for device closure.

\section{Case 2: MINOCA Secondary to Hypercoagulable State}

A 49-year-old man with a past medical history of sickle cell disease presented with chest pain. A 12-lead electrocardiogram showed normal sinus rhythm with no significant STsegment deviations. Cardiac troponin trended up to 11.5 $\mathrm{ng} / \mathrm{ml}$. Echocardiogram showed normal left ventricle function without significant regional wall motion abnormalities. Coronary angiography demonstrated normal coronary arteries. Cardiac MRI exhibited in the inferior wall myocardial edema on T2-weighted imaging (Fig. 2A), and a focal area of subendocardial late gadolinium enhancement, is suggestive of an acute myocardial infarction (Fig. 2B and C). A diagnosis of MINOCA secondary to microvascular obstruction was made, and the patient was discharged on antiplatelet therapy [45].

\section{Case 3: MINOCA Secondary to COVID-19}

A 79-year-old man with a past medical history of hypertension and hyperlipidemia presented with several days of shortness of breath, accompanied by blood-tinged sputum and productive cough, in the context of known exposure to COVID-19. Chest X-ray showed bilateral ground glass opacities. Nasopharyngeal PCR for COVID-19 was positive. He was admitted with a diagnosis of acute respiratory failure due to COVID-19 pneumonia and prescribed corticosteroids and

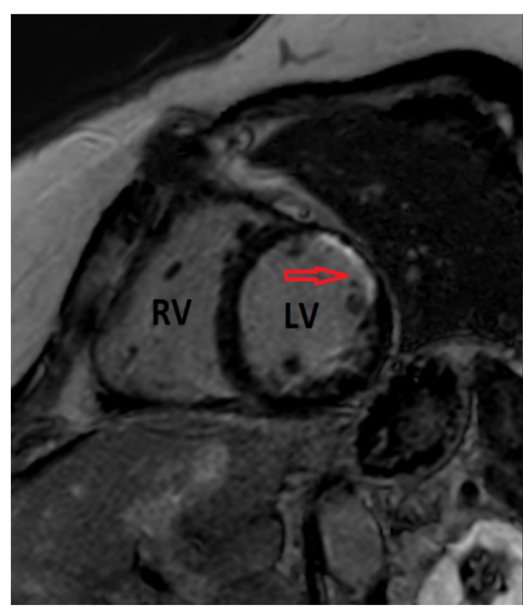

A

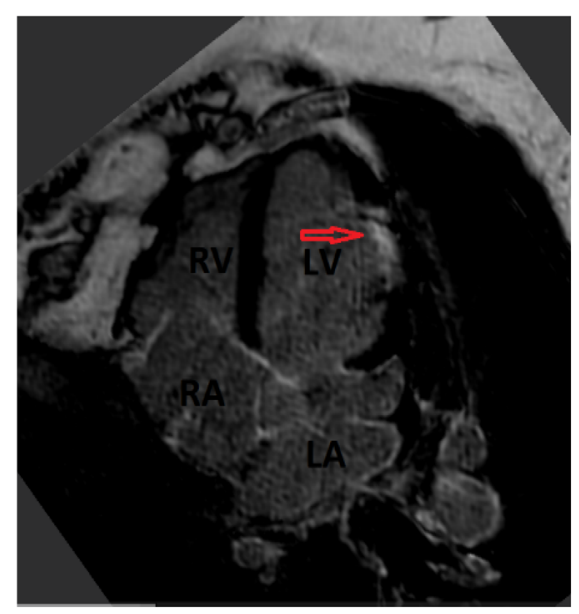

B

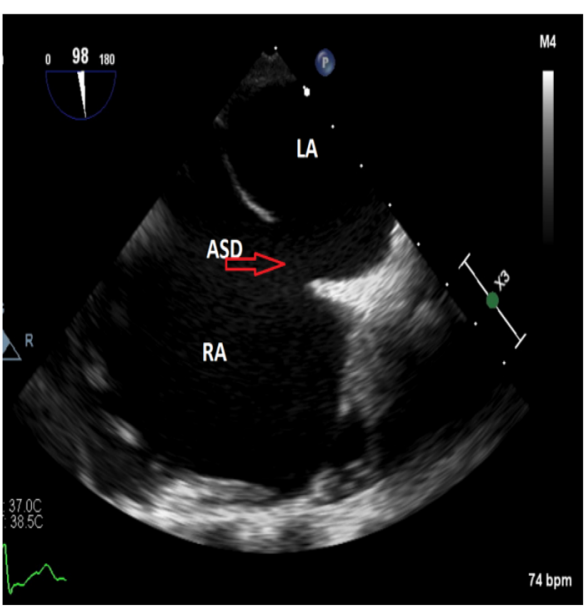

C
Fig. 1 A and B Cardiac MRI: post contrast images showed focal transmural myocardial infarction at the mid-ventricular portion of the anterolateral wall (red arrows point to scar in images $\mathbf{A}$ and $\mathbf{B}$ ). $\mathbf{C}$
Transesophageal echo revealed a small size secondary type atrial septal defect (red arrow points to atrial septal defect in image $\mathbf{C}$ ) 


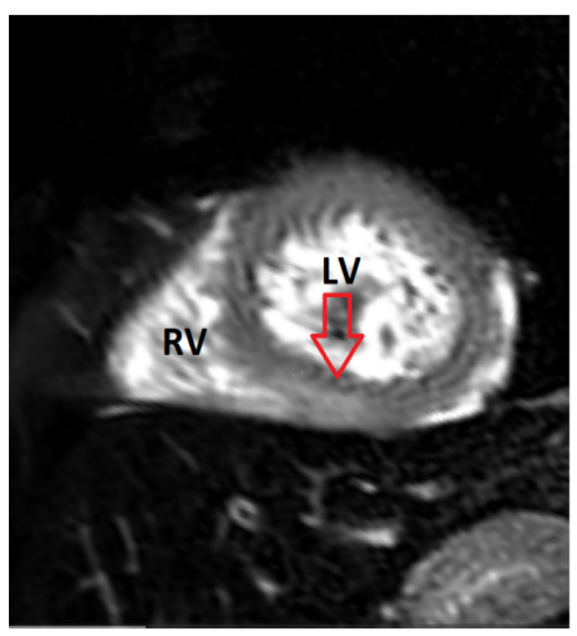

A

Fig. 2 A Cardiac MRI: T2-weighted images showed myocardial edema of the inferior wall, suggestive of an acute myocardial injury (red arrow points to myocardial edema in image A) (from Am J Med. 2020 Aug; 133(8):e425-e426) [42]. B and C Post contrast images showed a focal

antibiotics. On admission, his 12-lead electrocardiogram showed subtle ST elevations in leads I, II, and V4-V6 (Fig. 3A). Cardiac troponin peaked at $11 \mathrm{ng} / \mathrm{ml}$. At that time, he denied chest pain and cardiac catheterization was deferred, given his active COVID-19 infection. He was treated conservatively with heparin and dual antiplatelet therapy. Subsequent coronary CT angiography after recovery from COVID-19 and discharge exhibited nonobstructive coronary artery disease. A cardiac MRI performed 4 months after initial presentation showed normal left ventricle function and no evidence of myocardial edema (Fig. 3B) or myocardial scar (Fig. 3C).

Given the clinical presentation with ST elevation, it is likely that the patient had MINOCA, although other etiologies for an elevated troponin in a patient with COVID-19 infection must also be considered. Unfortunately, the MRI was performed at a time remote from the acute event and therefore a area of inferior subendocardial late gadolinium enhancement (red arrows point to scar in images B and C). (From Am J Med. 2020 Aug; 133(8):e425-e426) [42]

definitive diagnosis could not be obtained. This case highlights the vast possibilities in a patient with COVID-19 infection and suspected MINOCA and emphasizes the importance of timely assessment when feasible to establish an appropriate diagnosis including early cardiac MRI performed in the acute phase (less than 2 weeks).

\section{Conclusion}

MINOCA is not uncommon and has various etiologies. MINOCA is a syndrome that requires further investigation, and treatment should be tailored to the underlying cause. It is critical to use a systematic approach with multimodality imaging when treating patients with MINOCA to help determine the underlying cause and rule out MINOCA mimics. It is hoped that a more systematic approach to evaluating patients

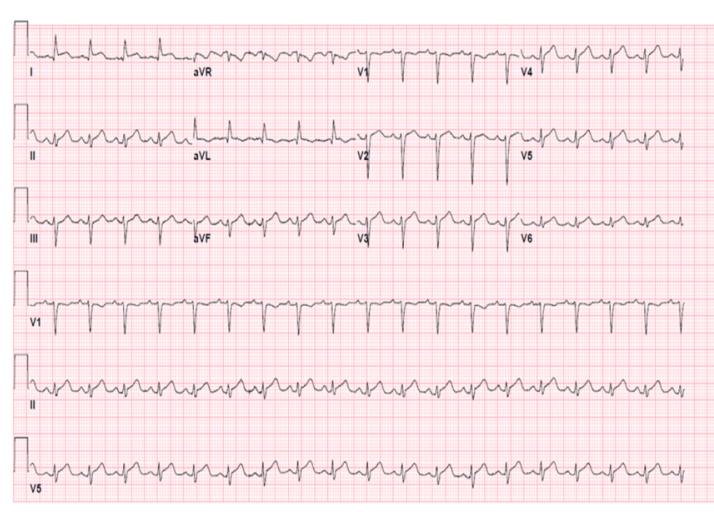

A

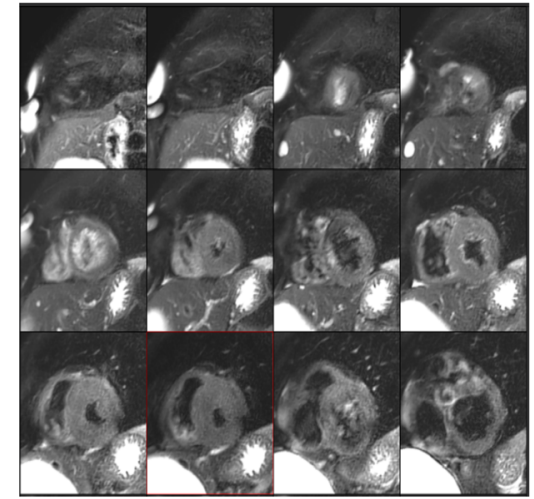

B

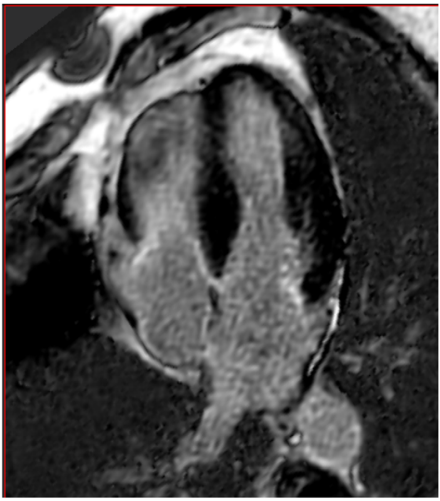

C

Fig. 3 A A 12-lead ECG showed subtle ST elevations in leads I, II, and V4-V6. B Cardiac MRI: T2-weighted images showed no evidence of myocardial edema. C Cardiac MRI: post contrast images showed no evidence of myocardial scar 
with MINOCA will result in better treatment and prognosis for these patients.

\section{Declarations}

Conflict of Interest The authors declare that they have no conflict of interest.

Human and Animal Rights and Informed Consent This article does not contain any studies with human or animal subjects performed by any of the authors.

\section{References}

Papers of particular interest, published recently, have been highlighted as:

•- Of major importance

1. Thygesen K, Alpert JS, Jaffe AS, Chairman BR, Bax JJ, Morrow DA, et al. Fourth Universal Definition of Myocardial Infarction (2018). Glob Heart. 2018;13:305-338.

2.• Tamis-Holland JE, Jneid H, Reynolds HR, Agewall S, Brilakis ES, Brown TM, et al. Contemporary diagnosis and management of patients with myocardial infarction in the absence of obstructive coronary artery disease: a scientific statement from the American Heart Association. Circulation. 2019;139:e891-908 The findings of this publication go into great detail about the various etiologies for MINOCA, as well as diagnostic workup and management.

3. Pasupathy S, Air T, Dreyer RP, Tavella R, Beltrame JF. Systematic review of patients presenting with suspected myocardial infarction and nonobstructive coronary arteries. Circulation. 2015;131:86170 .

4. Safdar B, Spatz E, Dreyer R, Beltrame J, Lichtman J, Reynolds H, et al. Presentation, clinical profile and prognosis of young patients with myocardial infarction with non-obstructive coronary arteries (MINOCA) - results from the variation in recovery: role of gender on outcomes of young AMI patients (VIRGO) study. J Am Heart Assoc. 2018;7.

5. Smilowitz NR, Mahajan AM, Roe MT, Hellkamp AS, Chiswell K, Gulati M, et al. Mortality of myocardial infarction by sex, age, and obstructive coronary artery disease status in the action registry-gwtg (Acute Coronary Treatment and Intervention Outcomes Network Registry-Get With the Guidelines). Circ Cardiovasc Qual Outcomes. 2017;10:e03443.

6. Jung RG, Parlow S, Simard T, Chen C, Ghataura H, Kishore A, et al. Clinical features, sex differences and outcomes of myocardial infarction with nonobstructive coronary arteries: a registry analysis. Coron Artery Dis. 2021;32:10-6.

7. Agewall S, Beltrame JF, Reynolds HR, Niessner A, Rosano G, Caforio AL, et al. ESC working group position paper on myocardial infarction with non-obstructive coronary arteries. Eur Heart J. 2017;38:143-53.

8. Collet JP, Thiele H, Barbato E, Barthélémy O, Bauersachs J, Bhatt DL, et al. 2020 ESC Guidelines for the management of acute coronary syndromes in patients presenting without persistent ST-segment elevation. Eur Heart J. 2021;42:1289-1367.

9. Talebi S, Moreno P, Dominguez AC, Tamis-Holland JE. The imaging toolbox to assess patients with suspected myocardial infarction in the absence of obstructive coronary artery disease (MINOCA). Curr Cardiol Rep. 2020;22:134.

10. Ouldzein H, Elbaz M, Roncalli J, Cagnac R, Carrie D, Puel J, et al. Plaque rupture and morphological characteristics of the culprit lesion in acute coronary syndromes without significant angiographic lesion: analysis by intravascular ultrasound. Ann Cardiol Angeiol (Paris). 2012;61:20-6.

11. Reynolds HR, Srichai MB, Iqbal SN, Slater JN, Mancini GB, Feit $\mathrm{F}$, et al. Mechanisms of myocardial infarction in women without angiographically obstructive coronary artery disease. Circulation. 2011;124:1414-25.

12.• Reynolds HR, Maehara A, Kwong RY, Sedlak T, Saw J, Smilowitz NR, et al. Coronary optical coherence tomography and cardiac magnetic resonance imaging to determine underlying causes of myocardial infarction with nonobstructive coronary arteries in women. Circulation. 2021;143:624-40 This study highlights the use of intracoronary imaging and advanced noninvasive imaging modalities to diagnose specific etiologies of MINOCA.

13. Saw J. Coronary angiogram classification of spontaneous coronary artery dissection. Catheterization and cardiovascular interventions. Catheter Cardiovasc Interv. 2014;84(7):1115-22.

14. Saw J, Aymong E, Mancini GB, Sedlak T, Starovoytov A, Ricci D. Nonatherosclerotic coronary artery disease in young women. Can J Cardiol. 2014;30:814-9.

15. Hayes SN, Kim ESH, Saw J, Adlam D, Arslanian-Engoren C, Economy KE, et al. Spontaneous coronary artery dissection: current state of the science: a scientific statement from the American Heart Association. Circulation. 2018;137:e523-57.

16. Pozo-Osinalde E, García-Guimaraes M, Bastante T, Aguilera MC, Rodríguez-Alcudia D, Rivero F, et al. Characteristic findings of acute spontaneous coronary artery dissection by cardiac computed tomography. Coron Artery Dis. 2020;31:293-9.

17. Lobo AS, Cantu SM, Sharkey SW, Grey EZ, Storey K, Witt D, et al. Revascularization in patients with spontaneous coronary artery dissection and ST-segment elevation myocardial infarction. J Am Coll Cardiol. 2019;74:1290-300.

18. Tweet MS, Eleid MF, Best PJ, Lennon RJ, Lerman A, Rihal CS, et al. Spontaneous coronary artery dissection: revascularization versus conservative therapy. Circ Cardiovasc Interv. 2014;7:777-86.

19. Saw J, Humphries K, Aymong E, Sedlak T, Prakash R, Starovoytov A, et al. Spontaneous coronary artery dissection: clinical outcomes and risk of recurrence. J Am Coll Cardiol. 2017;70:1148-58.

20. O'Gara PT, Kushner FG, Ascheim DD, Casey DE Jr, Chung MK, de Lemos JA, et al. 2013 ACCF/AHA guideline for the management of ST-elevation myocardial infarction: a report of the American College of Cardiology Foundation/American Heart Association Task Force on Practice Guidelines. Circulation. 2013; 127:e362-425.

21. Amsterdam EA, Wenger NK, Brindis RG, Casey DE Jr, Ganiats TG, Holmes DR Jr, et al. 2014 AHA/ACC guideline for the management of patients with non-ST-elevation acute coronary syndromes: a report of the American College of Cardiology/ American Heart Association Task Force on Practice Guidelines. Circulation. 2014;130:e344-426.

22. Montone RA, Niccoli G, Fracassi F, Russo M, Gurgoglione F, Camma G, et al. Patients with acute myocardial infarction and non-obstructive coronary arteries: safety and prognostic relevance of invasive coronary provocative tests. Eur Heart J. 2018;39:91-8.

23. Lee DH, Park TK, Seong CS, Gwag HB, Lim AY, Oh MS, et al. Gender differences in long-term clinical outcomes and prognostic factors in patients with vasospastic angina. Int J Cardiol. 2017;249: 6-11.

24. Prinzmetal M, Kennamer R, Merliss R, Wada T, Bor N. Angina pectoris. I. A variant form of angina pectoris; preliminary report. Am J Med. 1959;27:375-88. 
25. Kunadian V, Chieffo A, Camici PG, Berry C, Escaned J, Maas A, et al. An EAPCI expert consensus document on ischaemia with nonobstructive coronary arteries in collaboration with European Society of Cardiology Working Group on Coronary Pathophysiology \& Microcirculation endorsed by Coronary Vasomotor Disorders International Study Group. Euro Intervention. 2021;16:1049-69.

26. Guidelines for diagnosis and treatment of patients with vasospastic angina (coronary spastic angina) (JCS 2013). Circ J. 2014;78: 2779-801.

27. Slavich M, Patel RS. Coronary artery spasm:current knowledge and residual uncertainties. Int J Cardiol Heart Vasc. 2016;10:47-53.

28. Shibata T, Kawakami S, Noguchi T, Tanaka T, Asaumi Y, Kanaya $\mathrm{T}$, et al. Prevalence, clinical features, and prognosis of acute myocardial infarction attributable to coronary artery embolism. Circulation. 2015 Jul 28;132(4):241-50.

29. Stepien K, Nowak K, Wypasek E, Zalewski J, Undas A. High prevalence of inherited thrombophilia and antiphospholipid syndrome in myocardial infarction with non-obstructive coronary arteries: comparison with cryptogenic stroke. Int J Cardiol. 2019;290: $1-6$.

30. Rosendaal FR, Van Hylckama VA, Tanis BC, Helmerhorst FM. Estrogens, progestogens and thrombosis. J Thromb Haemost. 2003;1:1371-80.

31. Wahl A, Jüni P, Mono ML, Kalesan B, Praz F, Geister L, et al. Long-term propensity score-matched comparison of percutaneous closure of patent foramen ovale with medical treatment after paradoxical embolism. Circulation. 2012;125(6):803-12.

32.• Giustino G, Pinney SP, Lala A, Reddy VY, Johnston-Cox HA, Mechanick JI, et al. Coronavirus and cardiovascular disease, myocardial injury, and arrhythmia: JACC focus seminar. J Am Coll Cardiol. 2020;76:2011-23 This publication is one of the few available literatures which describe spectrum of cardiovascular complications seen in COVID-19 infection.

33. Bangalore S, Sharma A, Slotwiner A, Yatskar L, Harari R, Shah B, et al. ST-segment elevation in patients with Covid-19 - a case series. N Engl J Med. 2020;382:2478-80.

34. Imazio M, Klingel K, Kindermann I, Brucato A, De Rosa FG, Adler $\mathrm{Y}$, et al. COVID-19 pandemic and troponin: indirect myocardial injury, myocardial inflammation or myocarditis? Heart. 2020;106: $1127-31$.

35. Abou-Ismail MY, Diamond A, Kapoor S, Arafah Y, Nayak L. The hypercoagulable state in COVID-19: incidence, pathophysiology, and management. Thromb Res. 2020;194:101-15.

36. Pellegrini D, Kawakami R, Guagliumi G, Sakamoto A, Kawai K, Gianatti A, et al. Microthrombi as a major cause of cardiac injury in
COVID-19: a pathologic study. Circulation. 2021;143(10):103142.

37. Popovic B, Varlot J, Metzdorf PA, Jeulin H, Goehringer F and Camenzind $\mathrm{E}$. Changes in characteristics and management among patients with ST-elevation myocardial infarction due to COVID-19 infection. Catheter Cardiovasc Interv. 2021;97:E319-26.

38. Varga Z, Flammer AJ, Steiger P, Haberecker M, Andermatt R, Zinkernagel AS, et al. Endothelial cell infection and endotheliitis in COVID-19. Lancet. 2020;395:1417-8.

39. Kawakami R, Sakamoto A, Kawai K, Gianatti A, Pellegrini D, Nasr A, et al. Pathological evidence for SARS-CoV-2 as a cause of myocarditis: JACC review topic of the week. J Am Coll Cardiol. 2021;77(3):314-25.

40. Mahmud E, Dauerman HL, Welt FGP, Messenger JC, Rao SV, Grines C, et al. Management of acute myocardial infarction during the COVID-19 pandemic: a position statement from the Society for Cardiovascular Angiography and Interventions (SCAI), the American College of Cardiology (ACC), and the American College of Emergency Physicians (ACEP). J Am Coll Cardiol. 2020;76:1375-84.

41. Sadeghipour P, Talasaz AH, Rashidi F, Sharif-Kashani B, Beigmohammadi MT, Farrokhpour M, et al. Effect of intermediate-dose vs standard-dose prophylactic anticoagulation on thrombotic events, extracorporeal membrane oxygenation treatment, or mortality among patients with COVID-19 admitted to the intensive care unit: the INSPIRATION randomized clinical trial. JAMA. 2021:1842.

42. Fraissé M, Logre E, Pajot $\mathrm{O}$, Mentec H, Plantefève G, Contou D. Thrombotic and hemorrhagic events in critically ill COVID-19 patients: a French monocenter retrospective study. Crit Care. 2020;24: 275.

43. Meizlish ML, Goshua G, Liu Y, Fine R, Amin K, Chang E, et al. Intermediate-dose anticoagulation, aspirin, and in-hospital mortality in COVID-19: a propensity score-matched analysis. Am J Hematol. 2021;96:471-9.

44. Gerbaud E, Arabucki F, Nivet H, Barbey C, Cetran L, Chassaing S, et al. OCT and CMR for the diagnosis of patients presenting with MINOCA and suspected epicardial causes. JACC Cardiovasc Imaging. 2020;13:2619-31.

45. Lee S, Kaplin S, Tamis-Holland J, Talebi S. MINOCA in a patient with sickle cell disease. Am J Med. 2020 Aug;133(8):e425-6.

Publisher's Note Springer Nature remains neutral with regard to jurisdictional claims in published maps and institutional affiliations. 